\title{
Mecânica Clássica - Quadraturas
}

\author{
Mario Cezar Bertin ${ }^{1}$ \\ ${ }^{1}$ Instituto de Física, Universidade Federal da Bahia
}

September 28, 2020

\section{Energia Potencial em uma dimensão}

Na seção passada, estudamos o caso da partícula livre. Por não interagir com nenhum outro sistema físico, a partícula livre tem trajetória retilínea, uma vez escolhido um referencial inercial para analisá-la. Para descobrir a curva que representa o movimento, utilizamos o postulado da conservação da energia mecânica e o fato de que a partícula livre só possui energia cinética. Agora, vamos fazer com que esta partícula possa interagir com outros sistemas físicos, mas que ainda tenha seu movimento restrito a uma trajetória retilínea. Na prática, apenas tipos especiais de interação manterão uma partícula em uma única dimensão cartesiana. No entanto, vamos nos utilizar desta situação ideal para introduzir outros conceitos importantes.

Vamos supor, agora, um sistema composto de duas partículas de massas $m_{1}$ e $m_{2}$. A única interação no sistema é a interação entre as duas partículas, portanto, o sistema em si é isolado. Ambas as partículas permanecerão restritas a uma trajetória retilínea, portanto, a interação resulta em movimento unidimensional para cada partícula. Nesta configuração, a energia do sistema é composta pela energia cinética da partícula $1, K_{1}$, da energia cinética da partícula $2, K_{2}$, e da energia de interação $V_{12}=V_{21}=V\left(x_{1}, x_{2}\right)$, denominada energia potencial. No geral, a energia potencial pode depender das posições, velocidades e acelerações de ambas as partículas, mas no caso mais simples, vamos supor que dependa apenas da posição. Assim, temos

$$
E=K_{1}+K_{2}+V_{12}=\frac{1}{2} m_{1} v_{1}^{2}+\frac{1}{2} m_{2} v_{2}^{2}+V\left(x_{1}, x_{2}\right) .
$$

Aqui, os observáveis são vetores em uma dimensão, de modo que podemos ignorar a notação vetorial e trabalhar com o módulo desses vetores.

Se o sistema é isolado, esta energia se conserva no tempo, ou seja,

$$
\frac{d E}{d t}=0 \Longrightarrow\left(m_{1} a_{1}+\frac{\partial V}{\partial x_{1}}\right) v_{1}+\left(m_{2} a_{2}+\frac{\partial V}{\partial x_{2}}\right) v_{2}=0
$$

Como a trajetória é unidimensional, todos os vetores são colineares, assim, a equação acima pode ser satisfeita de várias formas. A conservação da energia não é suficiente neste caso, porque matematicamente o sistema não é unidimensional. A trajetória o é, mas precisamos de duas variáveis de posição para identificar um estado do sistema em determinado tempo $t$. Essas variáveis são as posições $x_{1} \mathrm{e} x_{2}$. Embora o movimento seja unidimensional, dizemos que ele possui dois graus de liberdade. Neste caso, precisaremos de mais um postulado para tratar deste caso, o que deixaremos para a próxima seção.

Contudo, uma generalização deste sistema pode ser tratada unidimensionalmente apenas com o sexto postulado, assumindo-se ignorância com relação a uma parte do sistema físico. Considere, por exemplo, uma partícula de massa $m$ restrita a uma trajetória retilínea, mas que interage com um sistema de partículas, chamado sistema 2. Vamos assumir que não conhecemos nada sobre o sistema interagente, ou seja, não temos 
informação de sua energia de movimento ou sua energia interna. A energia do sistema completo (partícula + sistema 2) deveria ser a soma:

1. Da energia cinética da partícula 1 ;

2. Da soma da energia cinética de todas as partículas constituintes do sistema 2 ;

3. Da interação de cada partícula do sistema 2 com a partícula 1 ;

4. Da interação interna das partículas do sistema 2.

Complicado. Mas em muitos sistemas de interesse é possível modelar a interação do sistema 2 com a partícula 1 com uma única energia potencial $V(x)$. Neste caso, estamos ignorando o que ocorre com o sistema 2 , já que não temos como ter informações completas sobre ele, e estamos analisando apenas o movimento da partícula 1. Neste caso, a energia relacionada à partícula 1 é dada apenas por

$$
E=\frac{1}{2} m v^{2}+V(x) .
$$

No geral, não esperamos que esta energia seja conservada, pois estamos deixando de fora o sistema 2. Assim, a partícula 1 passa a ser um sistema aberto, ou não isolado, portanto, o postulado 6 não se aplica. Mas existem sistemas nesta situação que possuem energia (1) conservada. São exemplos de sistemas conservativos.

Assim, o sistema descrito pela energia (1) é genuinamente unidimensional, possuindo um único grau de liberdade, representado pela posição $x$. Se esta energia se conserva, temos

$$
\frac{d E}{d t}=0 \Longrightarrow\left(m a+\frac{\partial V}{\partial x}\right) v=0,
$$

que resulta em

$$
m \ddot{x}=-\frac{\partial V}{\partial x} .
$$

equação (2) é o que conhecemos como a equação de movimento do sistema 1.

O fato é que é possível modelar a interação sobre um sistema físico de interesse, resultando da interação com um sistema desconhecido, em um grande número de aplicações. Este é o caso, por exemplo, ao analisarmos corpos em queda na Terra. Se o corpo em queda tem massa e dimensões muito menores que a Terra, ele pode ser modelado como uma partícula. A Terra, por outro lado, é um corpo extenso complexo, mas que pode ser modelado por um sistema de partículas, como o sistema 2 acima. Não é necessário conhecer a dinâmica da Terra para estudar a queda do corpo com um alto grau de precisão. Como a interação gravitacional, que rege a interação neste caso, é conservativa, podemos ignorar a Terra e modelar a queda do objeto como o movimento de uma partícula com energia potencial dependente apenas da posição do corpo com relação a uma posição de referência.

Assim, cada expressão para $V(x)$ remonta a um sistema conservativo unidimensional distinto. A partícula livre, tratada na seção passada, é um sistema de potencial zero, ou constante. Note que, se a energia potencial é constante, a energia cinética continua um invariante dinâmico. Como resultado, o acréscimo de um valor constante à energia de uma partícula não muda o movimento da partícula. No caso da partícula livre, basta ver que (2) se resume a $\ddot{x}=0$ se $V$ constante, portanto, um potencial constante não resulta em verdadeira interação.

\section{A quadratura}

Vamos supor, agora, o caso de uma partícula restrita a uma reta, sujeita a um potencial que depende apenas da posição dessa partícula na reta, ou seja, $V=V(x)$. A ausência da velocidade ou da aceleração pode parecer pouco geral, mas o fato é que muitos sistemas de interesse possuem $V$ como função apenas da coordenada. Neste caso, a energia da partícula é dada pela expressão (??). Por enquanto, vamos supor que esta energia 
se conserva. Assim, E é uma constante de movimento. Assim como no caso da partícula livre, cada valor de $E$ definirá uma curva distinta sobre a trajetória retilínea. Vejamos como isso ocorre.

Da expressão (3), vemos que

$$
v^{2}=\frac{2}{m}[E-V(x)] \Longrightarrow v= \pm \sqrt{\frac{2}{m}[E-V(x)]},
$$

que dá origem à equação diferencial total

$$
d x= \pm d t \sqrt{\frac{2}{m}[E-V(x)]} .
$$

Contudo, diferentemente do caso da partícula livre, o lado direito depende de $x$ em razão da energia potencial. Não é possível simplesmente integrar esta expressão. Mas se considerarmos que o movimento é retilíneo e que, em um subdomínio da curva $x=x(t)$, a função da curva seja bijetora, teremos, pelo menos para um intervalo $\Delta t$ definido, uma curva inversa $t=t(x)$ bem definida. Neste caso, podemos escrever

$$
d t= \pm \sqrt{\frac{m}{2}} \frac{d x}{\sqrt{E-V(x)}} .
$$

Caso a curva não tenha inversa única em determinado domínio, podemos quebrar o domínio em partes onde a função $t=t(x)$ é bem definida. Na expressão (4), vemos que o lado esquerdo depende apenas do tempo, enquanto o lado direito depende apenas da posição. Assim, podemos tomar a integral

$$
t-t_{0}= \pm \sqrt{\frac{m}{2}} \int_{x_{0}}^{x} \frac{d x^{\prime}}{\sqrt{E-V\left(x^{\prime}\right)}}
$$

A integral definida, denominada quadratura, pode ser resolvida analiticamente em poucos casos. Mas pode ser sempre trabalhada numericamente ou estimada por métodos aproximativos se a energia potencial for analítica. Vamos supor que, resolvida, a integral resulte em uma função de $x$. Assim, podemos escrever

$$
t-t_{0}=\Theta\left(x, E, x_{0}\right)
$$

em que explicitamos a dependência na energia e na posição inicial. Como $t=t(x)$ tem uma inversa por definição no domínio $I=\left[t_{0}, t\right]$, podemos inverter a expressão acima para obter a curva $x=\Theta^{-1}\left(x_{0}, t_{0}, E, t\right)$. Esta é a curva percorrida pela partícula com potencial $V(x)$ energia constante $E$ em uma dimensão. 


\section{O Potencial linear}

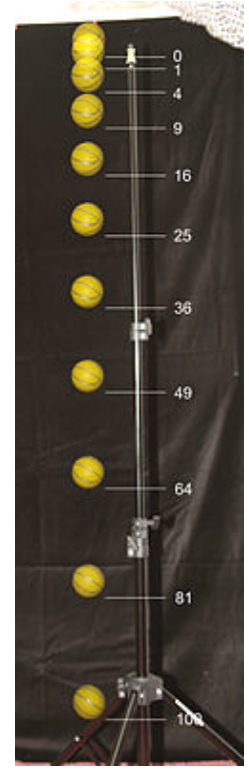

Figure 1: Exemplo de potencial linear: queda livre em baixa altitude. https://pt.wikipedia.org/wiki/ Queda_livre.

Vamos tratar do caso em que $V=-\alpha x$ com $\alpha \in \mathbb{R}_{+}$. Neste caso, temos a quadratura

$$
t-t_{0}= \pm \sqrt{\frac{m}{2}} \int_{x_{0}}^{x} \frac{d x^{\prime}}{\sqrt{E+\alpha x^{\prime}}}
$$

Podemos escrever a integral na forma

$$
\sqrt{\frac{m}{2}} \int_{x_{0}}^{x} \frac{d x^{\prime}}{\sqrt{E+\alpha x^{\prime}}}=\sqrt{\frac{m}{2 \alpha}} \int_{x_{0}}^{x} \frac{d x^{\prime}}{\sqrt{E / \alpha+x^{\prime}}} .
$$

Tomemos a transformação de variáveis $x^{\prime} \rightarrow y=E / \alpha+x^{\prime}$. Assim, $d x^{\prime}=d y$ e

$$
\sqrt{\frac{m}{2 \alpha}} \int_{x_{0}}^{x} \frac{d x^{\prime}}{\sqrt{E / \alpha+x^{\prime}}}=\sqrt{\frac{m}{2 \alpha}} \int_{E / \alpha+x_{0}}^{E / \alpha+x} y^{-1 / 2} d y
$$

O resultado vem a ser

$$
\sqrt{\frac{m}{2 \alpha}}\left(\frac{y^{1 / 2}}{1 / 2}\right)_{\frac{E}{\alpha}+x_{0}}^{\frac{E}{\alpha}+x}=\sqrt{\frac{2 m}{\alpha}}\left[\left(\frac{E}{\alpha}+x\right)^{1 / 2}-\left(\frac{E}{\alpha}+x_{0}\right)^{1 / 2}\right] .
$$

Então, temos

$$
t-t_{0}= \pm \sqrt{\frac{2 m}{\alpha}}\left[\left(\frac{E}{\alpha}+x\right)^{1 / 2}-\left(\frac{E}{\alpha}+x_{0}\right)^{1 / 2}\right] .
$$

Temos que inverter esta relação. Neste caso, escrevemos 


$$
\left(\frac{E}{\alpha}+x\right)^{1 / 2}= \pm \sqrt{\frac{\alpha}{2 m}}\left(t-t_{0}\right)+\left(\frac{E}{\alpha}+x_{0}\right)^{1 / 2} .
$$

Vamos elevar esta expressão ao quadrado:

$$
\frac{E}{\alpha}+x=\frac{E}{\alpha}+x_{0} \pm 2 \sqrt{\frac{1}{2 m}\left(E+\alpha x_{0}\right)}\left(t-t_{0}\right)+\frac{\alpha}{2 m}\left(t-t_{0}\right)^{2} .
$$

Como resultado:

$$
x=x_{0} \pm 2 \sqrt{\frac{1}{2 m}\left(E+\alpha x_{0}\right)}\left(t-t_{0}\right)+\frac{\alpha}{2 m}\left(t-t_{0}\right)^{2} .
$$

Note que esta curva tem a seguinte expressão para a velocidade:

$$
v=\frac{d x}{d t}= \pm 2 \sqrt{\frac{1}{2 m}\left(E+\alpha x_{0}\right)}+\frac{\alpha}{m}\left(t-t_{0}\right) .
$$

No tempo inicial $\mathrm{t}=\mathrm{t} 0$, temos

$$
v\left(t_{0}\right)= \pm 2 \sqrt{\frac{1}{2 m}\left(E+\alpha x_{0}\right)}=v_{0},
$$

que é a velocidade inicial. Ainda, temos a aceleração

$$
a=\frac{d v}{d t}=\frac{d^{2} x}{d t^{2}}=\frac{\alpha}{m} .
$$

Portanto, chegamos à já conhecida expressão para o movimento uniformemente variável

$$
x=x_{0} \pm v_{0} \Delta t+\frac{1}{2} a(\Delta t)^{2} .
$$

Note que o sinal \pm cobre os casos de velocidade inicial no sentido positivo ou negativo do eixo $\mathbf{e}_{1}$. Supostamente, poderíamos ter evitado trabalho ao simplesmente resolver a equação (eq:02) para o potencial $-\alpha x$. Mas isto certamente seria muito menos interessante para um curso avançado de mecânica. Além disso, a quadratura explicita a importância dos invariantes dinâmicos e suas relações com as curvas percorridas pelas partículas de forma mais fundamental. Esta consciência torna-se vital em sistemas mais complexos, como veremos. 


\section{O oscilador harmônico simples}
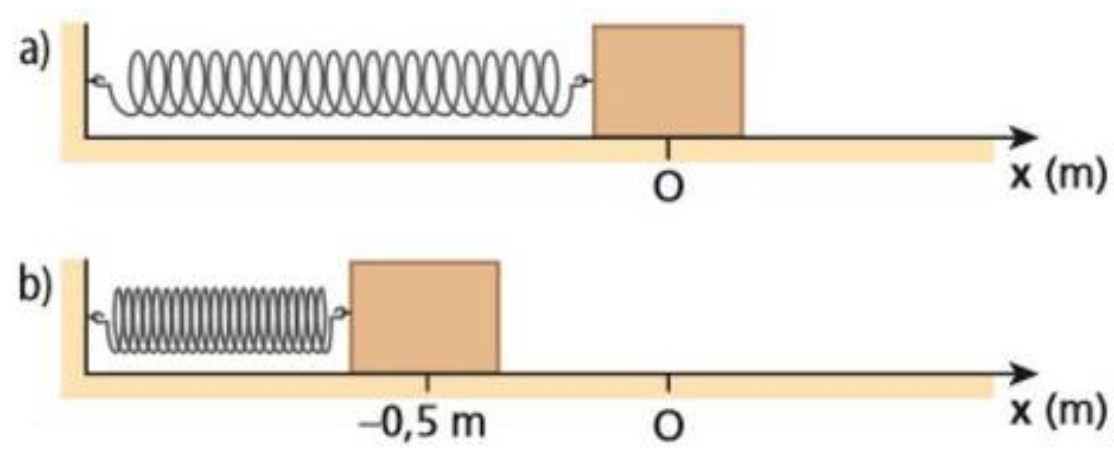

Figure 2: Exemplo de potencial quadrático: sistema massa-mola.

O próximo exemplo em ordem de complexidade é o potencial $V=\alpha x^{2}$, novamente para $\alpha$ real e não negativo. Vamos ver qual é sua curva solução através da quadratura

$$
t-t_{0}= \pm \sqrt{\frac{m}{2}} \int_{x_{0}}^{x} \frac{d x^{\prime}}{\sqrt{E-\alpha x^{\prime 2}}} .
$$

Nesta integral, é mais conveniente a forma

$$
t-t_{0}= \pm \sqrt{\frac{m}{2 E}} \int_{x_{0}}^{x} \frac{d x^{\prime}}{\sqrt{1-\frac{\alpha}{E} x^{\prime 2}}},
$$

visto que uma simples transformação de variáveis não resolve a integral.

Agora, introduzimos a transformação $x^{\prime} \rightarrow y=\sqrt{\frac{\alpha}{E}} x^{\prime}$, que resulta em,

$$
t-t_{0}= \pm \sqrt{\frac{m}{2 \alpha}} \int_{x_{0} \sqrt{\alpha / E}}^{x \sqrt{\alpha / E}} \frac{d y}{\sqrt{1-y^{2}}} .
$$

Esta é um integral que deve ser resolvida por uma transformação trigonométrica. A dica é a forma da raiz no denominador, $\sqrt{1-y^{2}}$. Se $y=\cos \theta$, temos que

$$
\sqrt{1-y^{2}}=\sqrt{1-\cos ^{2} \theta}=\sin \theta .
$$

Por outro lado, $d y=-\sin \theta d \theta$. Assim,

$$
\begin{aligned}
t-t_{0} a m p ; & = \pm \sqrt{\frac{m}{2 \alpha}} \int_{y_{0}}^{y} \frac{d y}{\sqrt{1-y^{2}}} \\
a m p ; & = \pm \sqrt{\frac{m}{2 \alpha}} \int_{\theta_{0}}^{\theta} \frac{-\sin \theta^{\prime} d \theta^{\prime}}{\sin \theta^{\prime}}= \pm \sqrt{\frac{m}{2 \alpha}} \int_{\theta_{0}}^{\theta} d \theta^{\prime}= \pm \sqrt{\frac{m}{2 \alpha}}\left(\theta-\theta_{0}\right) .
\end{aligned}
$$

Agora, temos que

$$
\theta=\arccos y=\arccos \left(\sqrt{\frac{\alpha}{E}} x\right)
$$


portanto,

$$
t-t_{0}= \pm \sqrt{\frac{m}{2 \alpha}}\left[\arccos \left(\sqrt{\frac{\alpha}{E}} x\right)-\arccos \left(\sqrt{\frac{\alpha}{E}} x_{0}\right)\right] .
$$

A inversa desta relação resulta em

$$
x=\sqrt{\frac{E}{\alpha}} \cos \left[\sqrt{\frac{2 \alpha}{m}} \Delta t \pm \arccos \left(\sqrt{\frac{\alpha}{E}} x_{0}\right)\right],
$$

que é o resultado final. Vamos ver o que há de familiar nesta equação.

Se $t=t_{0}$, podemos calcular a posição inicial da partícula:

$$
x\left(t_{0}\right)=\sqrt{\frac{E}{\alpha}} \cos \left[ \pm \arccos \left(\sqrt{\frac{\alpha}{E}} x_{0}\right)\right]= \pm \sqrt{\frac{E}{\alpha}} \sqrt{\frac{\alpha}{E}} x_{0}= \pm x_{0} .
$$

Este resultado seria esperado, a menos do sinal \pm . Vamos discutir isso em instantes.

A velocidade é calculada por

$$
v=\frac{d x}{d t}=-\sqrt{\frac{2 E}{m}} \sin \left[\sqrt{\frac{2 \alpha}{m}} \Delta t \pm \arccos \left(\sqrt{\frac{\alpha}{E}} x_{0}\right)\right],
$$

e a aceleração:

$$
a=-\frac{2}{m} \sqrt{\alpha E} \cos \left[\sqrt{\frac{2 \alpha}{m}} \Delta t \pm \arccos \left(\sqrt{\frac{\alpha}{E}} x_{0}\right)\right] .
$$

Com as soluções, torna-se claro que este sistema é um oscilador. Vamos supor que, na posição inicial $x_{0}$, o observador ligue seu relógio, de modo que $t_{0}=0$. Isto facilita nossa análise. Se $x(0)$ é positivo, temos a solução com sinal + em (??). Além disso, a função cosseno tem imagem no intervalo $[-1,1]$. Assim, o maior valor de $x$ é dado por $A \equiv \sqrt{E / \alpha}$, enquanto seu menor valor é dado por $A \equiv-\sqrt{E / \alpha}$. Portanto, $A$ é a amplitude do oscilador. Neste caso,

$$
x=A \cos \left[t \sqrt{\frac{2 \alpha}{m}} \pm \arccos \left(\frac{x_{0}}{A}\right)\right] .
$$

O tempo gasto para que o oscilador percorra um ciclo do movimento é denominado período. Podemos calcula-lo a partir da própria quadratura, visto que

$$
t-t_{0}=\sqrt{\frac{m}{2 \alpha}}\left[\arccos \left(\sqrt{\frac{\alpha}{E}} x\right) \pm \arccos \left(\sqrt{\frac{\alpha}{E}} x_{0}\right)\right] .
$$

Neste caso, o período é duas vezes o tempo que o oscilador gasta para percorrer o trajeto de $x=A$ a $x=-A$. Assim,

$$
\begin{aligned}
\text { Tamp } & ;=2 \sqrt{\frac{m}{2 \alpha}}\left[\arccos \left(-\sqrt{\frac{\alpha}{E}} A\right) \pm \arccos \left(\sqrt{\frac{\alpha}{E}} A\right)\right] \\
\text { amp } & ;=2 \sqrt{\frac{m}{2 \alpha}}[\arccos (-1) \pm \arccos (1)]=2 \pi \sqrt{\frac{m}{2 \alpha}} .
\end{aligned}
$$

Por outro lado, a frequência da oscilação é definida por $f=1 / T$, ou seja, $f=(\sqrt{2 \alpha / m}) /(2 \pi)$.

Ainda, a frequência angular, ou velocidade angular é definida por $\omega=2 \pi f$, que resulta em

$$
\omega=\sqrt{\frac{2 \alpha}{m}}
$$


Com estes novos parâmetros, temos as soluções

$$
\begin{aligned}
& \text { xamp } ;=A \cos \left[\omega t \pm \arccos \left(\frac{x_{0}}{A}\right)\right], \\
& \text { vamp } ;=-\sqrt{\frac{2 E}{m}} \sin \left[\omega t \pm \arccos \left(\frac{x_{0}}{A}\right)\right], \\
& \text { aamp } ;=-\frac{2}{m} \sqrt{\alpha E} \cos \left[\omega t \pm \arccos \left(\frac{x_{0}}{A}\right)\right] .
\end{aligned}
$$

Para completar esta seção, vamos calcular a energia em função de $\omega$ e $A$. Neste caso,

$$
E=\alpha A^{2}=\frac{1}{2} m \omega^{2} A^{2}
$$

Assim,

$$
\begin{aligned}
& x a m p ;=A \cos \left[\omega t \pm \arccos \left(\frac{x_{0}}{A}\right)\right], \\
& v a m p ;=-\omega A \sin \left[\omega t \pm \arccos \left(\frac{x_{0}}{A}\right)\right], \\
& a a m p ;=-\omega^{2} A \cos \left[\omega t \pm \arccos \left(\frac{x_{0}}{A}\right)\right],
\end{aligned}
$$

como esperado. No geral, temos essas expressões escritas para a condição inicial $x_{0}=A$, o que resulta em

$$
x=A \cos (\omega t), v=-\omega A \sin (\omega t), a=-\omega^{2} A \cos (\omega t) .
$$

Observando as soluções (??), vemos claramente que $a=-\omega^{2} x$, ou seja,

$$
\ddot{x}+\omega^{2} x=0 \text {. }
$$

Esta é uma equação diferencial ordinária de segunda ordem, e é definida como a equação de movimento do sistema. Assim, uma equação de movimento em mecânica clássica geralmente é uma EDO de segunda ordem, salvo nos sistemas que estudaremos no formalismo hamiltoniano. 